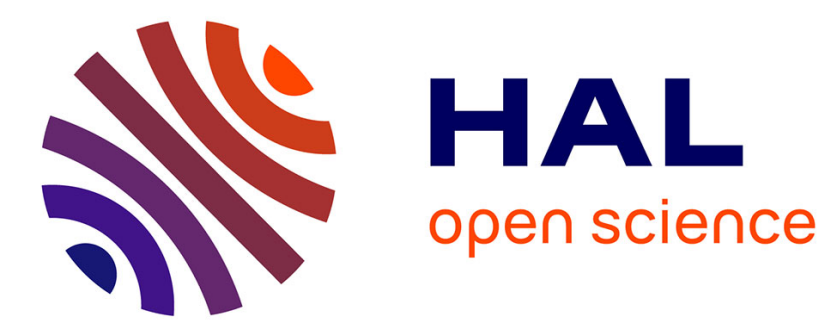

\title{
A Semantic Model to Query Spatial-Temporal Data
} Benjamin Harbelot, Helbert Arenas, Christophe Cruz

\section{To cite this version:}

Benjamin Harbelot, Helbert Arenas, Christophe Cruz. A Semantic Model to Query Spatial-Temporal Data. The 6th International Workshop on Information Fusion and Geographic Information Systems: Environmental and Urban Challenges, May 2013, St. Petersburg, Russia. hal-00779463

\section{HAL Id: hal-00779463 https://hal.science/hal-00779463}

Submitted on 22 Jan 2013

HAL is a multi-disciplinary open access archive for the deposit and dissemination of scientific research documents, whether they are published or not. The documents may come from teaching and research institutions in France or abroad, or from public or private research centers.
L'archive ouverte pluridisciplinaire HAL, est destinée au dépôt et à la diffusion de documents scientifiques de niveau recherche, publiés ou non, émanant des établissements d'enseignement et de recherche français ou étrangers, des laboratoires publics ou privés. 


\title{
A Semantic Model to Query Spatial-Temporal Data
}

\author{
Benjamin Harbelot, Helbert Arenas, and Christophe Cruz \\ Laboratoire Le2i, UMR-6302 CNRS,Departement Informatique Dijon,University of \\ Burgundy 7 Boulevard Docteur Petitjean, BP 17867, 21078 Dijon CEDEX, France \\ \{benjamin.haberlot, helbert.arenas\}@checksem.fr \\ christophe.cruz@u-bourgogne.fr \\ http://checksem.u-bourgogne.fr
}

\begin{abstract}
There is a growing need for the study of spatial-temporal objects and their relationships. A common approach for this task is the use of relational databases, which unfortunately do not allow inference. In this research we introduce a new approach that uses the concept of "continuum" together with ontologies and semantic web technologies. The "continuum" allows us to define parent-child relationships between representations of objects. It also allows us to compare the evolution of two different objects and establish the relationships between them along time. Our approach is based on the 4D fluent, which is extended to obtain spatial-temporal qualitative information from the analysis of objects and their relationships. The results of our analysis are later added to our knowledge base enhancing it. Our preliminary results are promising and we plan to further develop the model in the near future.
\end{abstract}

Keywords: GIS, semantics, spatio-temporal

\section{Introduction}

There is a growing demand for tools to handle spatial-temporal information, however currently there is a limited number of available options. This research introduces a novel approach that adds semantics to spatial-temporal data, allowing reasoning and inference. When designing a spatial temporal information system it is necessary to deal with three aspects of the data: 1) The spatial aspect which consists of geometry, 2) the temporal aspect which defines the interval of existence of the geometries and finally 3) the semantic aspect of an object, which aims to provide with a meaning beyond the purely geographic [1]. There is a fourth optional feature which that consists in the representation of the semantics derived from the evolution of the various attributes of the objects. For instance in the study of land parcels, we found that each one has a id, a geometry and possible other attributes such as for instance land use, which might evolve along time. By considering the evolution of the geometry and attribute values we would be able to handle the semantics resulting from changes in the objects and in the relationships among them along time. 
Currently among the GIS tools there is a lack of suitable options when there is need to perform spatial-temporal analysis. There are previous examples in the field of RDBMS for storage of dynamic spatial objects. However RDBMS do not implement inference or reasoning mechanisms. Ontologies on the other hand allow these mechanisms by creating formal representations of concepts, properties and relationships between concepts. Traditionally ontologies have been applied to static domains, in the sense that entities represented in these ontologies do not change over time or space. However spatial objects are dynamic; it is possible an object changes their attributes and spatial representation along time. Objects with an spatial representation might grow, shrink, change their shape, split, disappear or merge into a new object. To overcome this limitation in this paper we propose a model that develops the concept of continuum within an ontology. The continuum is an abstract concept that represents the evolution of an object over time from spatial and semantic points of view. The continuum allows the representation of objects with known semantics and spatial representations with a defined lifetime. Using our model we can register the object evolution and link an object with its spatial representation that corresponds to any given point of time. The continuum model allows the handling of spatial-temporal dynamics and stores the process in the form of a spatial graphs.

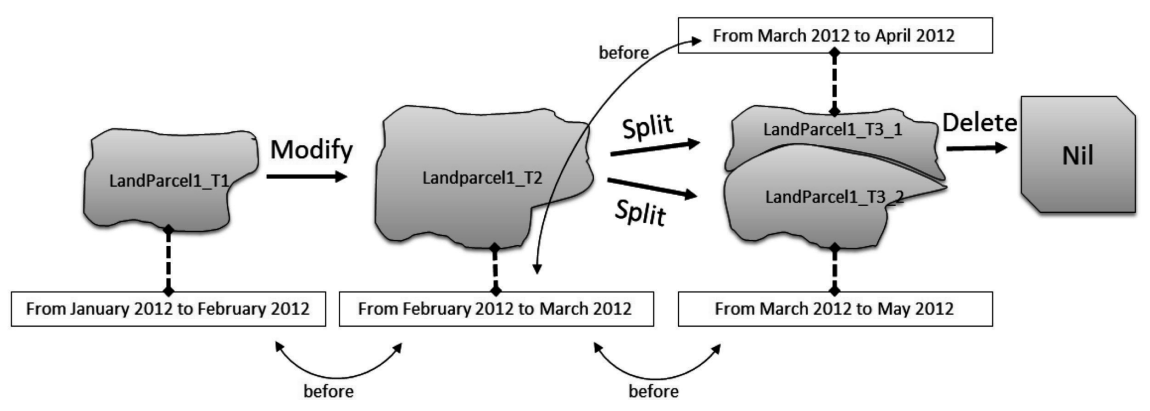

Fig. 1. Evolution of a land parcel over time

We can distinguish two approaches to handle the semantics of the spatialtemporal objects: 1) Adding semantic capabilities to GIS tools, which are not necessarily ontologies and 2) Inserting spatial data into an ontology. The first approach provides only an extension of the attributes, while the second allows the description of the knowledge in an enhanced way that can be used to perform reasoning on spatial data. Our work is based on the second approach.

Several methodologies for the dynamic representation of objects and their properties have already been proposed. Among the most well known are the temporal description logic, temporal RDF, versioning, reification, N-ary relationships and the 4D-fluent approach. 
The 4D-fluent approach can effectively represent temporal information in OWL. Other works such as SOWL are based on this approach, extending OWL and allowing it to handle not only quantitative temporal information but also qualitative one. Qualitative temporal information like "before" or "after" have the advantage of representing relationships between time intervals with unknown starting or ending points. Qualitative information can be inferred from quantitative one. Qualitative information can also be used as an alternative if there is missing quantitative information. The inference of qualitative information from quantitative one, both spatial and temporal is the core of this work. To accomplish this goal a set of SWRL rules are defined and used to enrich the ontology with inferred facts. Additionally SWRL provides a powerful extension that allows the definition of its own methods. These methods are called built-ins. In our work we have developed built-ins that perform calculations between space and temporal representations. The result of the operations is included in our ontology to enrich it. Besides the inference capabilities a system of this kind must also provides mechanisms to query the knowledge base. Traditionally, the query language used for ontologies has been SPARQL. However because it can only be used with RDF based ontologies, has a limited effectiveness. An alternative to SPARQL is SQWRL, which retains the semantics of OWL. SQWRL syntax is similar to SQL, providing an easy and explicit way to query the system.

Work on knowledge representation is presented in Section 2. In Section 3 we present the proposed model and the inference system implemented. Finally in Section 4 we present the query language chosen for this work.

\section{Background}

The most common way to represent spatially dynamic objects is through the use of Relational DBMS. A very interesting example is DOMINO which uses a combination of ArcView and Informix as an application server to store spatial data and ArcIMS as a render tool. Another interesting example is SECONDO, a prototype of a DBMS that is able to handle moving objects. SECONDO is able to store the history of movements of a given object and respond to spatial temporal queries [2] [3]. However one limitation of these examples is that although they are able to represent dynamic objects, they are not able to perform any inference or reasoning with the stored information. On the other hand we have the semantic web technologies that have been designed expressly to represent knowledge in a form that can allow the use of reasoning and inference mechanisms. Several representation languages have been defined in the Semantic Web.In this section, we discuss the existing data management technologies in a scalable ontology.

\subsection{Representing spatial dynamics in an ontology}

The two main philosophical theories concerning the object persistence over time are: endurantism and perdurantism. The first one, endurantism, considers objects as three dimensional entities that exist wholly at any given point of their 
life. On the other hand, perdurantism, also known as the four dimensional view, considers that objects have temporal parts, "timeslices" that compose their temporal dimension[4]. This approach represents the different properties of an object over time as fluent. A fluent is a property valid only during certain intervals or moments in time. In this research we use a perdurantism approach to record the changes and movement of spatial objects over time. In order to apply this approach within an ontology is necessary to convert static properties into dynamic ones. In the field of conceptual modelling, this is expressed in the concept-class association defined between two entities. OWL has limitations for the representation of dynamic entities; it only allows binary relations between individuals. In order to solve this limitation previous research have proposed several alternatives such as: temporal RDF, reification, versioning, or 4D-fluent. Temporal RDF proposes an extension of the standard RDF for naming properties with the corresponding time interval, allowing explicit management of time. [5]. Reification is a technique used to represent n-ary relations with languages such as OWL which allow only binary relations [6] . Versioning is described as the ability to handle changes in ontologies by creating and managing multiple variants of them [7]. However these methods have some disadvantages. Temporal RDF relies solely on RDF triples, therefore it does not have all the expressiveness of OWL, for instance using only RDF is not possible to express qualitative relations. Reification allows the use of a triplet as object or subject of a property. But this method has also its limitations, for instance the transformation from a static property into a dynamic one increases substantially the complexity of the ontology, reducing the querying and inference capabilities. Additionally reification is prone to redundant objects which reduces its effectiveness. The major drawback of Versioning, is the redundancy generated by the slightest change of an attribute. Any information requests must be performed on multiple versions of the ontology, reducing its effectiveness. The 4D-fluent approach is based on the perdurantism philosophical approach. It considers that objects have a temporal dimension composed by several temporal representations, each corresponding to a defined interval of time. Together all those representations compose the temporal dimension of an object. In the literature 4D-fluent is the most well known method to handle dynamic properties in an ontology. It has a simple structure allowing to easily transform a static ontology into a dynamic one. However it is not possible using this approach to handle explicit semantics. This fact causes two problems: 1) it is difficult to maintain a close relationship between geometry and semantics; and 2) it increases the complexity for querying the temporal dynamics and understanding the modelled knowledge. Furthermore, this approach does not define qualitative relations to describe the type of change that has occurred or to describe the temporal relationships between objects. We cannot then know which objects have undergone a change and what objects might be the result of that change.

The spatial evolution of an object involves movement or a change of shape [8]. In the case of a movement, it is easy to identify and locate the object before and after the event. However when an object suffers a succession of changes a 
key question arises: how much can an object change before its semantics are modified? And if there is a semantic change, then how do we know that this is the same object at different times? The $4 \mathrm{D}$ fluent approach does not allow an object to change its nature, only allows the change of the value of some of its properties. However the semantics associated with a geometry may change. For example a land parcel may change from being forest into being an urban parcel. In this example the geometry has not changed, however there is a semantic change. Conversely, the semantics might not change as the geometry evolves. For instance, a given urban land parcel might expand by purchasing neighbouring parcels. In the first example (see figure $2 \mathrm{~A}$ ), there are two different semantic objects associated with the same geometry at different times. In the second example (see figure 2B), we have two related geometries for the same semantic object at different times.
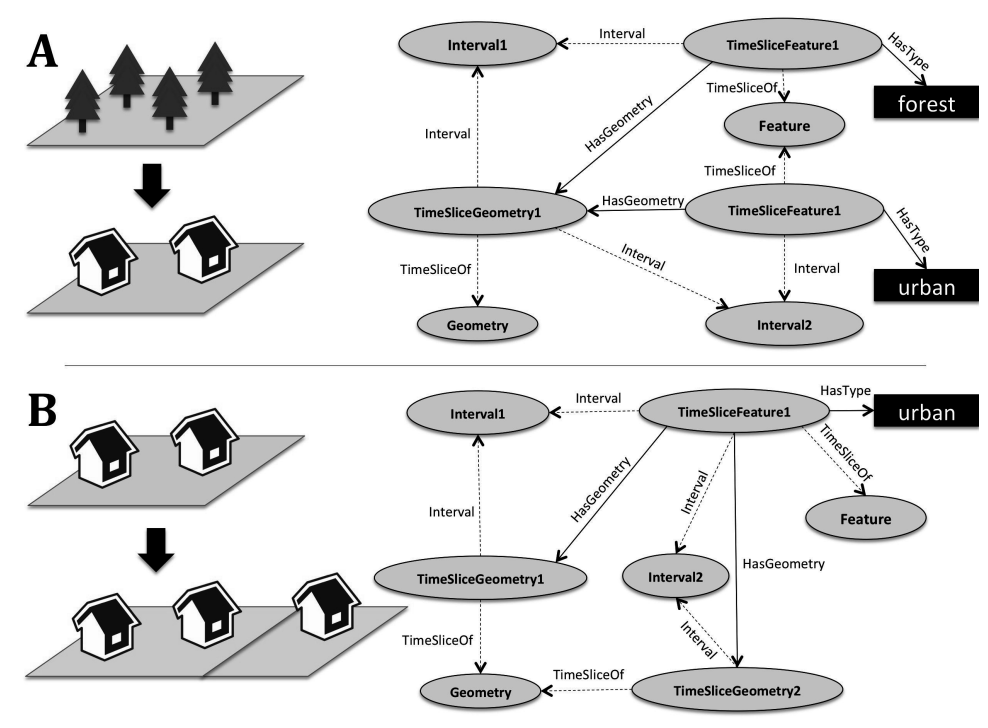

Fig. 2. Examples of the evolution A) Two different semantic objects for the same geometry. B) Two related geometries for the same semantic object.

\subsection{Representation of semantic relations}

To study the evolution of a concept over time we require tools to define temporal concepts in an ontology. The OWL-Time ontology is used for this purpose to describe the temporal aspect of content of web pages and properties of web services. Moreover, this ontology provides good support for expressing topological relationships between times and time intervals, as well as times and dates [9]. Another tool designed to handle temporal concepts in an ontology is SWRL 
Temporal Ontology. This tool has all the capabilities of OWL-Time ontology and additionally has the advantage of built-ins, which can be used to perform calculations between intervals and time instants to infer relationships from qualitative and quantitative information [10].The qualitative relations in the time domain are based on binary and mutually exclusive relations as proposed by Allen [11]:

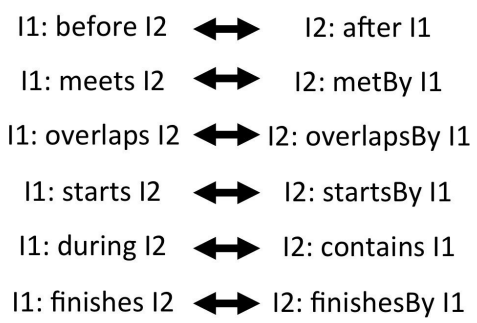

11: equals 12

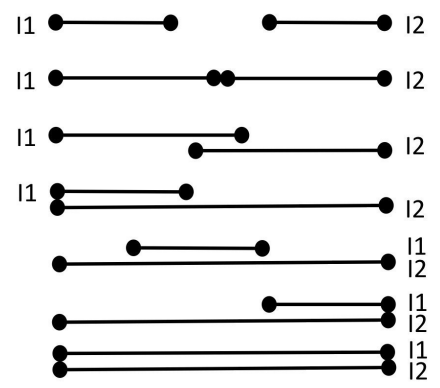

Fig. 3. Allen temporal relations

The addition of Allen relations can increase the expressive power of the system by adding qualitative information in addition to the quantitative one. Allen relationships allow to go even further when intervals are semi closed (just a defined start date or just a defined end date) [12]. For example, lets suppose we have three intervals I1, I2 and I3. We know that I1 meets I2 and that I2 contains I3, we do not know the ending point of I2, or the starting point of I3. However Then we can infer that because I2 contains I3, I3 must be after I1, even if the information about start and ending points is incomplete (See figure 4). Lack of knowledge caused by semi closed intervals is largely filled by the integration of Allen relations to the model.

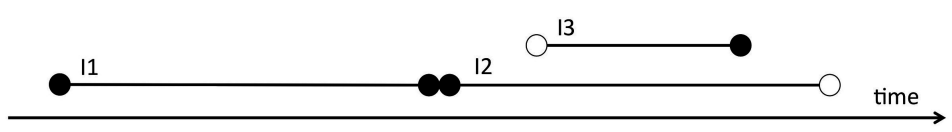

Fig. 4. Using Allen temporal relations to infer new knowledge

In GIS, objects are represented by points, lines, polygons or other more complex figures based on these geometries. All these geometries are defined using the coordinates of points which are quantitative information. The use of an ontology is of interest when one wants to study the relationships between these objects. There are mainly three types of relationships between geometries: directional, 
metric and topological relationships. The relationships based on quantitative information can be translated into qualitative data [8], in a similar fashion as we have described for the temporal aspect. By analysing a set of moments and time intervals it is possible to deduce qualitative topological relationships between objects. The topological analysis between two objects is done using the models: Dimensionally Extended Nine-Intersection Model (DE-9IM) or RCC8 [13]. In both cases, we obtain an equivalent set of topological relationships for specific regions. To calculate the spatial relationships between two geometries DE-9IM model takes into account the inside, and outside of the contour of the geometries leading to the analysis of nine intersections as depicted in table 1 .

Table 1. DE-9IM Intersection Matrix

$\begin{array}{llll} & \operatorname{dim}(I(A) \bigcap I(B)) & \operatorname{dim}(I(A) \bigcap B(B)) & \operatorname{dim}(I(A) \bigcap E(B)) \\ D E-9 I M(A, B)= & \operatorname{dim}(B(A) \bigcap I(B)) & \operatorname{dim}(B(A) \bigcap B(B)) & \operatorname{dim}(B(A) \bigcap E(B)) \\ & \operatorname{dim}(E(A) \bigcap I(B)) & \operatorname{dim}(E(A) \bigcap B(B)) & \operatorname{dim}(E(A) \bigcap E(B)) \\ & I=\text { interior } & B=\text { Boundary } & E=\text { Exterior }\end{array}$

Table 2 depicts the eight possible spatial relationships resulting from DE9IM.

Table 2. Topological predicates and their corresponding meanings

\begin{tabular}{ll}
\hline Topological & Predicate Meaning \\
\hline Equals & The Geometries are topologically equal. \\
Disjoint & $\begin{array}{l}\text { Geometries have no point in common. } \\
\text { Intersects }\end{array}$ \\
Touches & $\begin{array}{l}\text { Geometries have at least one point in common (inverse of Disjoint). } \\
\text { points). }\end{array}$ \\
Crosses & $\begin{array}{l}\text { Geometries share some but not all interior points, and the dimension of } \\
\text { the intersection is less than that of at least one of the Geometries. }\end{array}$ \\
Overlaps & $\begin{array}{l}\text { Geometries share some but not all points in common, and the intersection } \\
\text { has the same dimension as the Geometries themselves. }\end{array}$ \\
Within & $\begin{array}{l}\text { Geometry A lies in the interior of Geometry B. } \\
\text { Contains }\end{array}$
\end{tabular}

\subsection{Reasoning with spatial-temporal information in an ontology}

Only a reasoning system allows the switch from quantitative to qualitative data. In [14] the authors introduce a model in which spatial-temporal information contained in a database and a spatial-temporal inference system work together. However, no information is given on the Semantic Web technologies, only the Java language is quoted as a component of the inference engine, therefore the 
universality and effectiveness of the inference system can be questioned. Another work is [15] in which the authors propose a reasoning system that combines the topological calculus capabilities of a GIS and the inference capabilities of the semantic web field. However the notion of time is not incorporated into this model. Research conducted by [12] shows the model-based approach that uses SOWL 4D-fluent and ontology OWL-Time. A set of SWRL rules is established to allow the system to infer the topological, relational and metric relations. A customized language was developed by the authors for this model similar on structure to SQL. The SOWL language allows simple spatial-temporal querying for both static and dynamic data.

\subsection{Querying information on a spatial-temporal ontology}

Traditionally, the SPARQL has been the query language in ontologies. It is a W3C recommendation that operates at the level of RDF graphs. However using SPARQL the queries become relatively complex for spatial-temporal information. An extension of this language, st-SPARQL, defines new functions that allow it to handle geometries but not temporal data [16]. St-SPARQL is based on an extension of RDF called st-RDF that integrates contact geometries and incorporate time in RDF. St-SPARQL and SPARQL are both based on RDF graphs therefore their inference capabilities are limited. Other research led to the development of SOWL, a language designed for querying models based on $4 \mathrm{D}$ time-fluent. SOWL is easy to use because of its structure similar to SQL. It provides specific spatial-temporal topology operators besides orientation and metric ones.

\section{The Continuum Model}

The continuum model represents objects with three distinct aspects as depicted in figure $5 \mathrm{~A}$ :

Semantics: To identify an object and describe its associated knowledge.

Space: It is the graphical representation of the object.

Time: It represents the interval or time instants that describe the temporal existence of the object.

The continuum model aims to follow the evolution of dynamic objects. In the model spatial, temporal and semantic aspects of the object are handled independently.Each change automatically creates a new object. If the change occurs only on the spatial part of the object, the newly created object will retain the same semantics as the original one and vice versa if the change occurs only on the semantic part.

Each change adds to a genealogy of spatial-temporal objects. Some objects can then be defined as "parent" and / or "child" of other spatial-temporal objects. This genealogy enforces a coherency between the time intervals of each spatial-temporal object. Figure 5B depicts an example of objects genealogy. In 


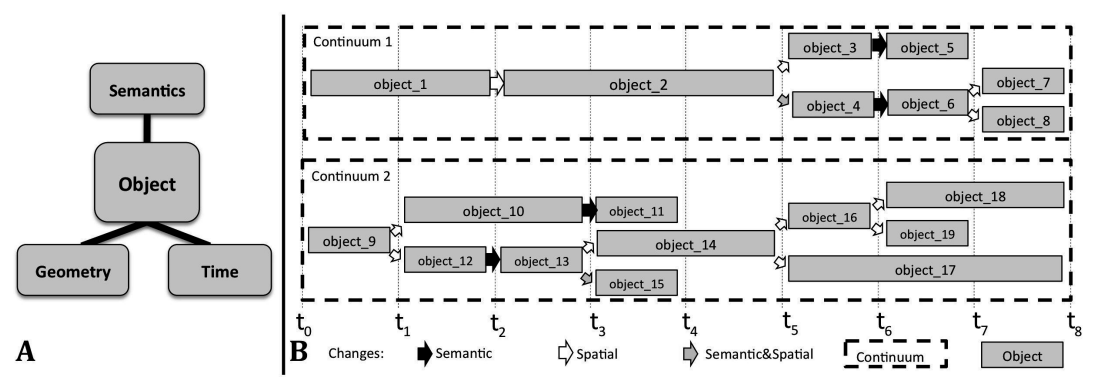

Fig. 5. A) The three components of an object within the continuum model. B) Using the continuum model to represent the evolution of an entity

this example "object_2" is the result of a spatial change on "object_1", then "object_2" is child of "object_1", then it is also true that the time interval of "object_1" meets the time interval of "object_2". In addition, "object_3" is the child of "object_2" then we can infer that the time interval of "object_3" is after the time interval of "object_1". Coherence between time intervals and genealogy can be verified by the system. In Figure 5B the arrows between the objects represent the established offspring relationships generated by the evolution of a real world object. It is possible to characterize the evolution of each object in the model according to the conceptual hierarchy depicted in Figure 6.

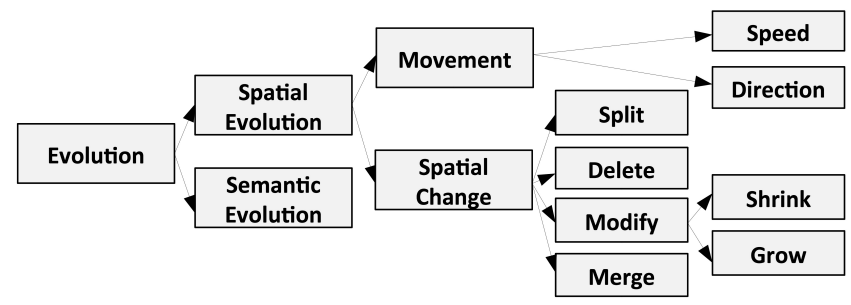

Fig. 6. Qualification of transition in the spatial graph.

A continuum is a set of representations of an entity along time, each representation has a valid, finite time interval. The model links each representation to its context. A representation can belong to more than one continuum, then continuums can intersect.Figure 5B depicts the evolution of an entity and how the continuum concept is used to study it.

But the relevance of the continuum model is not limited to this aspect. The objects represented in a continuum have a temporal part defined by a time interval, and a spatial part defined by a geometry. It is possible to analyse the qualitative relationships between representations of objects of two different continuums. 


\subsection{Rules for an appropriate use of the model}

The continuum model aims to observe and store the spatial-temporal evolution of entities over time. A dynamic entity can undergo two types of evolutions: a change or a movement. To allow the observation and recording of the evolution of an entity the model creates a new representation of the entity each time it suffers a change. We represent dynamics in a shopping center to illustrate our point.

For this example we have created a representation of a real shopping center in our spatial database. Later semantic and temporal information have been added and organized in the ontology. Figure 7 depicts the ontology class hierarchy. The main classes are "Continuum" and "siteFeature". The last one represents objects (semantically organized in class and subclass). The "Geometry" and "validTime" classes represent respectively the spatial and temporal parts of an object. The "spatialAnalysisResult" class is a special class which stores the results of "Union", "Difference", "Buffer" or "Intersection" spatial operators. More details about the implementation are depicted in Figure 8.

A shopping center is composed by stores. Each store has an owner. Within the mall, some stores appear, or disappear. A store might buy a neighbouring property then merge with it and grow, others might split and give rise to new stores, finally some shops might change their owners. In the mall, there are moving customers that enter and exit from the stores. The mall, and the stores which composed it, are considered as entities undergoing changes while customers are seen as moving entities. Spatial-temporal moving objects, such as customers walking through the mall, are manageable in the continuum model. The movement of a client is represented by its recorded position at regular time intervals. Each position has a spatial part corresponding to its representation in the geographic information system and a temporal part corresponding to the instant at which time this position was recorded. The continuum then aggregates all positions of a client hierarchically in time. The continuum model offers several options for managing the evolution of the mall. The user can decide to follow the evolution of the entity "mall" in which case it will be able to identify the emergence, disappearance or renovations that involve mall sections. Alternatively the user can follow the evolution of individual stores. It is also possible to combine both options and have a complete view of the mall evolution. Ultimately, for changing objects, the use of continuum depends on the goal the user has in mind. The continuum model offers different views of the same geographical region. The continuum model is very flexible and allows the handling of fixed and moving entities.

\subsection{Reasoning on the Continuum Model}

Reasoning capabilities are essential components of the application. They allow both to check the consistency of information and also to enrich the ontology automatically from data inferred through concepts such as transitivity, symmetry or inverse. The inference is done by introducing a set of SWRL rules. The use 


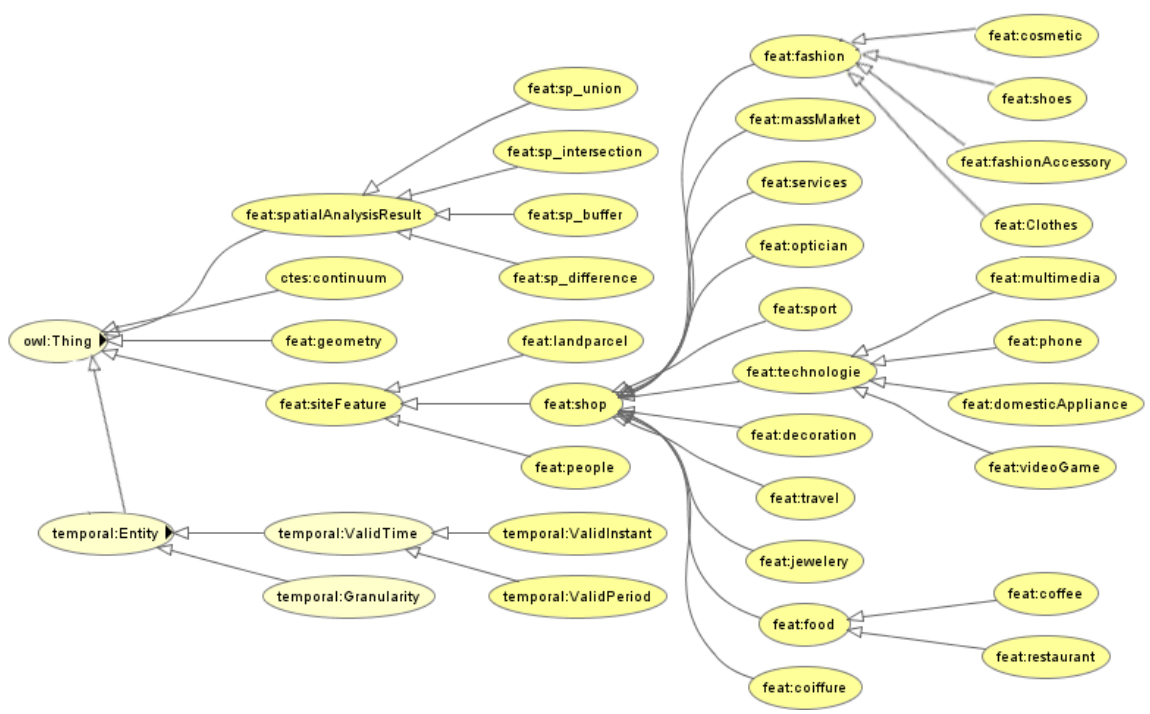

Fig. 7. Class hierarchy of the ontology (using protégé plug-in OWL viz-Tab)

of SWRL is crucial because it provides a powerful extension that allows the definition of customized methods, called built-ins.

Spatial Built-ins: In space, there are eight possible spatial relationships between two geometries. As explained above, the geometries are stored in a GIS system, and spatial analysis of these can be done in the GIS system. But the calculation of topological spatial relationships should be done in the ontology and more precisely with the SWRL rules. Spatial built-ins have been implemented to compute the topology between two geometries. The definition of these built-ins require an interconnection between the ontology and GIS which is done through a JAVA program. Spatial built-ins can be used with all the concepts represented in the ontology that have a spatial part. The topological calculation requires access to the geometry store in the GIS. When a spatial built-in is used in a SWRL rules, the JAVA program detects it and runs the calculation in the GIS.

Example: Give all the people within a given shop

feat : shop $(? x) \wedge$ feat : people $(? y) \wedge$ spatialswrlb :Within $(? x, ? y) \rightarrow$ sqwrl : $\operatorname{select}(? x, ? y)$

Temporal Built-ins: On the time domain, the Allen relationships are defined between time intervals. Temporal built-ins do not require any calculation but only provide a qualitative result from a comparison between intervals or instants.

Example: Give all shops existing on the 1st January of 2012

feat : $\operatorname{shop}(? x) \wedge$ temporal : hasValidTime $(? x, ?$ time 1$) \wedge$ temporal : contains(?time1,"2012-01-01") $\rightarrow$ sqwrl : select(?x) 


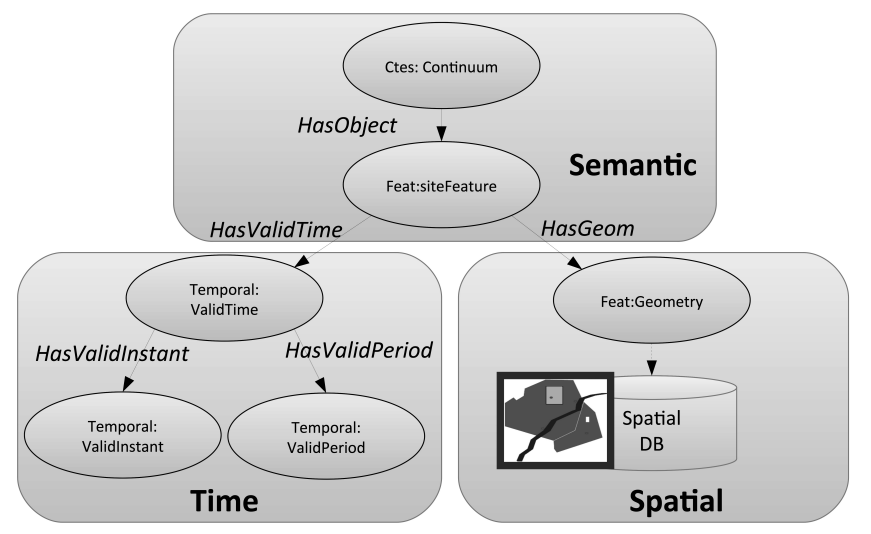

Fig. 8. Implementation of the Continuum Model in the ontology

Spatial-temporal Built-ins: There are no specific spatial-temporal built-ins, however the dynamic combination of spatial and temporal built-ins in a SWRL rule allows a spatial-temporal analysis between two objects. It may, for example, launch a topological analysis between spatial objects with only a common or disjoint time interval.

\section{Querying on the continuum model}

Apart from the SWRL built-ins explained above, there is a query language in SWRL, based on built-ins, called SQWRL [17]. This is a language specially adapted to OWL that retains all the expressiveness and semantics of OWL, contrary to SPARQL. SQWRL is a concise language, easily understood and semantically robust making it the ideal candidate to query a system whose inferences capabilities are a major issue. Its explicit SQL-like structure allows a good understanding for users unfamiliar with the Semantic Web domain.

Example A: Lets assume we have the positions of the customers for a certain mall. In this example we desire to identify all people that have entered into a shop that existed in December 2011. We would also like to know the number of recorded positions for each person in each store (see figure $9 \mathrm{~A}$ ):

feat : shop $(?$ shop $) \wedge$ temporal : hasValidTime $(?$ shop, ?time 1$)$

$\wedge$ temporal : contains $(?$ time 1, "2011 - 12") $\wedge$ feat : people(?people 1$)$

$\wedge$ temporal : hasValidTime(?people1,?time2)

$\wedge$ temporal : contains (?time1,?time 2$) \wedge$ sa: hasWithin $(?$ shop, ?people 1$)$

$\wedge$ isObject(?people1, ?continuum) o sqwrl : makeSet(?total,?people1)

$\wedge$ sqwrl: groupBy(?total,?shop, ?continuum) o sqwrl : size(?size,?total)

$\rightarrow$ sqwrl : select(?shop,?continuum,?people 1, ?size)

Example B: Lets assume we require to identify people that have meet in the restaurant (see figure $9 \mathrm{~B}$ ):

feat : people(?people1) ^temporal : hasValidTime(?people1,?time1) 

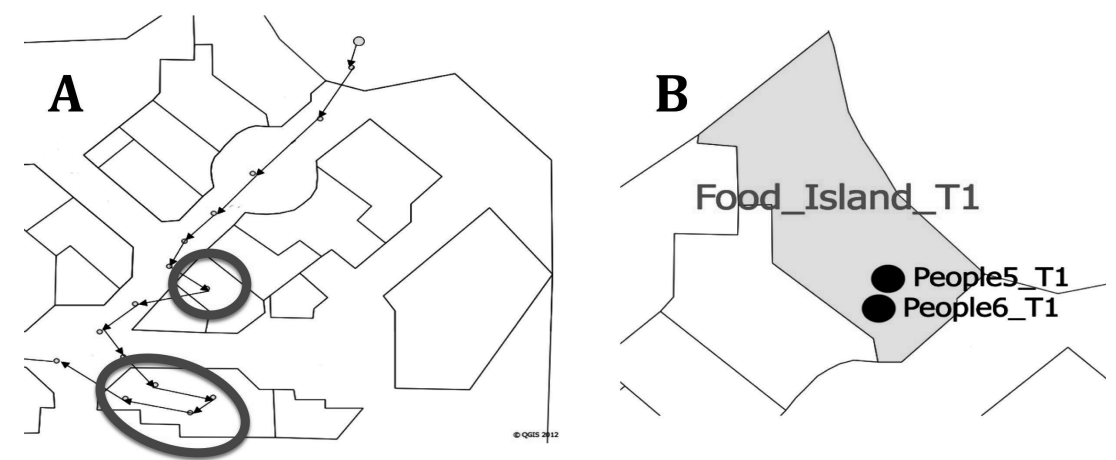

Fig. 9. Examples A and B

$\wedge$ temporal : hasValidTime(?people $2, ?$ time 2$)$

$\wedge$ temporal : equals(?time1,?time2, temporal : Minutes)

$\wedge$ abox : hasClass(?people 2, feat : people $) \wedge$ sa : isWithin(?people1,?restaurant)

$\wedge$ sa: isWithin(?people 2, ? restaurant $) \wedge$ abox : hasClass $(? z$, feat $:$ food $)$

$\wedge$ temporal : hasValidTime(?restaurant,?time 3$)$

$\wedge$ temporal : contains(?time3,?time 1$)$

$\wedge$ temporal : contains $(?$ time $3, ?$ time 2$) \wedge$ tbox : notEqualTo(?people1,?people 2$)$

$\rightarrow$ sqwrl : select(?people1,?people 2 , ?restaurant)

\section{Conclusions}

We introduce a model capable of handling temporal, spatial and spatial-temporal information in an ontology. The continuum model is based on the 4D-fluent and develops the continuum concept in the context of a spatial-temporal GIS. The model preserves understandable semantics for the dynamic objects represented.

The continuum model includes a set of rules and built-ins for inferring qualitative relations from quantitative data. It handles time and space independently for each object allowing the inclusion or not of time and space in queries of spatial, temporal or spatial-temporal nature.

Currently the semantics introduced in the system allow the identification of related objects along time.

This model introduces a novel approach for the handling of properties and attributes for each object. The semantic management of the properties and attributes for each object will be part of further research in order to develop a complete system for the semantics of spatial-temporal information.

\section{References}

1. Yuan, M.: Use of a three-domain representation to enhance GIS support for complex spatial-temporal queries. Transactions in GIS 3 (March 1999) 137-159 
2. SECONDO Team: SECONDO An extensible database system. (Online) http://dna.fernuni-hagen.de/Secondo.html/ (2012) (Accessed on November 2012).

3. Innerebner, M., Bhlen, M., Timko, I.: A web-enabled extension of a spatio-temporal DBMS. In: GIS '07 Proceedings of the 15th annual ACM international symposium on Advances in geographic information systems. (2007) 34:1-34:8

4. Welty, C., Fikes, R.: A reusable ontology for fluents in OWL. In: Proceedings of 2006 conference on Formal Ontology in Information Systems (FOIS 2006). (2006) $226-236$

5. Gutierrez, C., Hurtado, A., Vaisman, A.: Introducing time into RDF. IEEE Transactions on Knowledge and Data Engineering 19 (February 2007) 207-218

6. Hayes, P.: RDF semantics, W3C Recomendation, 10 february 2004. (Online) http://www.w3.org/TR/rdf-mt/ (2004) (Accessed on November 2012).

7. Klein, M., Fensel, D.: Ontology versioning on the Semantic Web. In: Proceedings of the First International Semantic Web Working Symposium SWWS'01. (July 2001) 75-91

8. Brisaboa, N., Mirbel, I., Pernici, B.: Constraints in spatio-temporal databases: A proposal for classification. In: Proceedings of the 3th. International Workshop on Evaluation of Modeling Methods in System Analysis and Design. (1998)

9. Hobbs, J., Pan, F.: Time ontology in OWL. (Online) http://www.w3.org/TR/owltime/ (Accessed on November 2012).

10. O'Connor, M., Das, A.: A method for representation and querying temporal information in OWL. In: Proceedings of Biomedical Engineering Systems and Technologies BIOSTEC 2010. (2010) 97-110

11. Allen, J.: Maintaining knowledge about temporal intervals. Communications of the ACM 26(11) (November 1983) 832-843

12. Batsakis, S., Petrakis, E.: SOWL: Spatio-temporal representation reasoning and querying over the semantic web. In: Proceedings of the 6th. International Conference on Semantic Systems I-SEMANTICS 2010. (September 2010) 15:1-15:9

13. Strobl, C.: Dimensionality Extended Nine-Intersection Model (DE-9IM). In: Encyclopedia of GIS Springer. Springer (2008) 240-245

14. Ryu, K., Ahn, Y.: Application of moving objects and spatiotemporal reasoning (2001) A TIMECENTER Technical Report.

15. Karmacharya, A., Cruz, C., Boochs, F., Marzani, F.: Integration of spatial processing and knowledge processing through the semantic web stack. In Claramunt, C., Levashkin, S., Bertolotto, M., eds.: GeoSpatial Semantics. Volume 6631 of Lecture Notes in Computer Science. Springer Berlin Heidelberg (2011) 200-216

16. Koubarakis, M., Kyzirakos, K.: Modeling and querying metadata in the semantic sensor web: The model stRDF and the query language stSPARQL. In Aroyo, L., Antoniou, G., Hyvnen, E., Teije, A., Stuckenschmidt, H., Cabral, L., Tudorache, T., eds.: The Semantic Web: Research and Applications. Volume 6088 of Lecture Notes in Computer Science. Springer Berlin Heidelberg (2010) 425-439

17. O'Connor, M., Das, A.: SQWRL: a query language for OWL. In: Proceedings of OWL: Experiences and Directions (OWLED) 6th International Workshop. (2009) 